\title{
BMJ Open Carbohydrate intake, obesity, metabolic syndrome and cancer risk? A two-part systematic review and meta-analysis protocol to estimate attributability
}

\author{
B Sartorius, ${ }^{1,2}$ K Sartorius, ${ }^{1,2,3}$ C Aldous, ${ }^{2,4}$ T E Madiba, ${ }^{2,4}$ C Stefan, ${ }^{2,5}$ T Noakes $^{6}$
}

To cite: Sartorius B, Sartorius K, Aldous C, et al. Carbohydrate intake, obesity, metabolic syndrome and cancer risk? A two-part systematic review and metaanalysis protocol to estimate attributability. BMJ Open 2016;6:e009301.

doi:10.1136/bmjopen-2015009301

- Prepublication history and additional material is available. To view please visit the journal (http://dx.doi.org/ 10.1136/bmjopen-2015009301).

BS and KS are joint first authors.

Received 3 July 2015 Revised 17 October 2015 Accepted 23 October 2015

CrossMark

For numbered affiliations see end of article.

Correspondence to Professor B Sartorius; Sartorius@ukzn.ac.za

\section{ABSTRACT}

Introduction: Linkages between carbohydrates, obesity and cancer continue to demonstrate conflicting results. Evidence suggests inconclusive direct linkages between carbohydrates and specific cancers.

Conversely, obesity has been strongly linked to a wide range of cancers. The purpose of the study is to explore linkages between carbohydrate intake and cancer types using a two-step approach. First the study will evaluate the linkages between carbohydrate intake and obesity, potentially stratified by metabolic syndrome status. Second, the estimated attributable fraction of obesity ascribed to carbohydrate intake will be multiplied against obesity attributable fractions for cancer types to give estimated overall attributable fraction for carbohydrate versus cancer type.

Methods and analysis: We will perform a comprehensive search to identify all possible published and unpublished studies that have assessed risk factors for obesity including dietary carbohydrate intake. Scientific databases, namely PubMed MEDLINE, EMBASE, EBSCOhost and ISI Web of Science will be searched. Following study selection, paper/data acquisition, and data extraction and synthesis, we will appraise the quality of studies and risk of bias, as well as assess heterogeneity. Meta-weighted attributable fractions of obesity due to carbohydrate intake will be estimated after adjusting for other potential confounding factors (eg, physical inactivity, other dietary intake). Furthermore, previously published systematic reviews assessing the cancer-specific risk associated with obesity will also be drawn. These estimates will be linked with the attributability of carbohydrate intake in part 1 to estimate the cancer-specific burden that can be attributed to dietary carbohydrates. This systematic review protocol has been developed according to the 'Preferred Reporting Items for Systematic review and Meta-Analysis Protocols (PRISMA-P) 2015'.

Ethics and dissemination: The current study will be based on published literature and data, and, as such, ethics approval is not required. The final results of this two part systematic review (plus multiplicative calculations) will be published in a relevant international peer-reviewed journal.

Trial registration number: PROSPERO CRD42015023257.
Strengths and limitations of this study

- To the best of our knowledge, the quantified influence of high carbohydrate intake on cancer, via obesity, has not been assessed using a meta-approach.

- This study will attempt to address this gap in an area of major importance that also includes a renewed focus on the controversy surrounding dietary options.

- One of the main possible limitations of this review could be the scarcity of studies/data on the subject. Cancer related to high carbohydrate intake in the absence of obesity will not be assessed due to the confounding effect of multiple variables and the lack of literature to quantify this effect.

\section{BACKGROUND}

Global estimates in 2005 indicated 937 million people were overweight and 328 million were obese. ${ }^{1}$ On the basis of GLOBOCAN estimates, about 14.1 million new cancer cases and 8.2 million deaths occurred in 2012 worldwide. The occurrence of cancer is increasing because of the growth and ageing of the population, as well as an increasing prevalence of established risk factors such as smoking, overweight, physical inactivity, and changing reproductive patterns associated with urbanisation and economic development. ${ }^{2}$ Emerging evidence also indicates that diet-related cancers are increasing in many developed countries as they progress to Western lifestyles and diets. ${ }^{34}$ The direct linkage between carbohydrates and cancer outcomes is inconclusive, and limited to a few types of cancer. Furthermore, the role of carbohydrates and population-level obesity is also unclear. Conversely, numerous meta-studies indicate obesity as a risk factor for a wide range of specific cancers. A majority of systematic reviews, however, are also disaggregated to 
either assess the relationship between carbohydrates and specific cancers, ${ }^{5-9}$ or obesity and cancer. ${ }^{10-14}$ The objective of this study is to investigate the relationship between carbohydrates and cancer via the mediating impact of obesity, taking into account the confounding role of metabolic syndrome. In particular, the study will first perform a meta-analysis to determine to what extent carbohydrates are associated with obesity and, second, determine the extent to which carbohydrates increase the risk of specific cancer outcomes via their association with obesity taking into account the presence of metabolic syndrome. In order to do this, a series of systematic reviews that have already investigated the relationship between obesity and specific cancers will be selected in order to develop a multiplicative attributable factor for each study that quantifies the association between carbohydrates and specific cancers.

\section{Carbohydrates and cancer}

The direct linkages between carbohydrates and cancer continue to demonstrate mixed results. Past systematic reviews suggest, rather than verify, potential linkages between carbohydrates (or different types of carbohydrates) and specific cancers. Examples of these relationships suggest a link between sucrose and colorectal cancer; lactose and ovarian cancer; ${ }^{5}$ fructose and pancreatic cancer; ${ }^{8}$ glycaemic load/glycaemic index and breast and gallbladder cancer, ${ }^{15}$ as well as colorectal and endometrial cancer. ${ }^{6}$ Conversely, another meta study found no relationship between carbohydrates and gastrointestinal and colorectal cancer risk. ${ }^{7}$ Very little (or no) evidence has been collected to show any risk between carbohydrates and cancers of the oral cavity, pharynx, stomach, lung, cervix, prostate and kidney. ${ }^{5}$ Considerable evidence has been offered to explain why carbohydrates can influence non-alcoholic fatty liver disease, non-alcoholic steatohepatitis and hepatocellular carcinoma (HCC) cancer. Although it is widely acknowledged that (most) cancers have a multivariable aetiology, ${ }^{16}$ a common explanation of the role of carbohydrates involves their direct relationship with the metabolic syndrome (eg, hyperinsulinaemia) and its resultant effect on the stimulation of insulin-like growth factors 1 , cell proliferation and the deregulation of apoptosis. ${ }^{6} 1617$ Despite these plausible explanations, no convincing epidemiological evidence supports the direct link between carbohydrates and HCC. ${ }^{9}$ Far more evidence exists to show that dietary fibre has a protective effect on a wide range of cancers, ${ }^{5} 1819$ rather than assessing the relationship between high carbohydrate intake and increased risk of cancer.

\section{Carbohydrates and obesity}

Body weight is ultimately determined by the interaction of genetic, environmental and psychosocial factors, acting through the physiological mediators of energy intake and expenditure. ${ }^{20}{ }^{21}$ Recent risk assessments have shown that there is no consistent association between the proportions of energy consumed as carbohydrate and body weight, and reinforce the dominance of total energy intake as the primary determinant of body weight. However, they have highlighted evidence that different types of carbohydrate, such as sugar-sweetened beverages (SSBs), have specific effects on the risk of obesity. ${ }^{22}$ An increasing number of systematic reviews have investigated the relationship between low versus high carbohydrate restricted calorie diets and their impact on body weight. In general, low carbohydrate restricted calorie diets have shown that they induce at least the same level (or higher) of weight loss than their low fat counterpart diets. ${ }^{1} 2324$ Low carbohydrate diets also substantially reduce body weight, body mass index (BMI), abdominal circumference, systolic and diastolic blood pressure, and triglycerides, as well as fasting glucose, glycated haemoglobin, plasma insulin and plasma $\mathrm{C}$ reactive protein, in addition to increasing high-density lipoprotein. ${ }^{25}$ Dietary carbohydrates are associated with weight gain ${ }^{26}$ and specific carbohydrates, such as SSBs, are positively associated with weight gain and obesity. ${ }^{27}$ From a physiological perspective, low carbohydrate diets may decrease calorie intake because they increase demands on protein and amino acid turnover for gluconeogenesis, which has a high energy cost. Alternatively, low carbohydrate diets may induce weight loss due to reducing insulin concentrations, thus promoting free fatty acid mobilisation from body fat storage. $^{28}$

\section{Obesity and cancer}

Obesity has been overwhelmingly implicated in the aetiology and projection of cancer incidence. ${ }^{2} 2930$ Obesity has been strongly linked to colon, renal, gallbladder, pancreatic, endometrial and postmenopausal breast cancers, as well as to malignant melanoma, oesophageal adenocarcinoma and leukaemia. ${ }^{5}{ }^{10}$ More specific meta-analysis shows that increased BMI is associated with many specific cancers, for example, renal cell cancer, ${ }^{31}$ breast cancer, ${ }^{32}$ biliary tract cancer, ${ }^{11}$ large B-cell lymphoma, ${ }^{33}$ endometrial cancer, ${ }^{12}$ thyroid cancer, ${ }^{34}$ colorectal cancer ${ }^{14}$ and meningioma. ${ }^{13}$ Although obesity is directly associated with metabolic syndrome and its proposed relationships with cancer, obesity directly results in the remodelling of tissue leading to an increase in adipose tissue. The increase in adipose tissue is directly associated with higher levels of inflammation and an increase in oxidative stress. ${ }^{17}$

\section{OBJECTIVES}

The purpose of this study is to explore the linkages between dietary carbohydrate intake and cancer outcomes, using a two-step approach, that is, mediated via obesity. First, the study will use a systematic review/ meta-analysis to evaluate the linkages between dietary carbohydrate intake and obesity, stratified by metabolic syndrome status. Second, the estimated population 
attributable fraction (PAF) of obesity ascribed to carbohydrate intake will be multiplied against the obesity versus cancer-type specific effect measures and PAF to give estimated overall PAF for carbohydrate versus cancer type.

\section{REVIEW QUESTION}

This systematic review will seek to address the following research question:

What fraction of specific cancers can be attributed to high carbohydrate intake through the mediating effect of obesity taking into account the effect of the metabolic syndrome?

\section{METHODS/DESIGN}

\section{Part 1: carbohydrates and obesity}

Registration of protocol with PROSPERO

In accordance with the guidelines, the systematic review protocol for part 1 has been registered with the International Prospective Register of Systematic Reviews (PROSPERO) on 8 June 2015 (registration number CRD42015023257).

This systematic review will be aligned to the Preferred Reporting Items for Systematic reviews and Meta-Analyses Protocols' (PRISMA-P) guidelines, ${ }^{35}$ to ensure all necessary steps have been followed (see online supplementary appendix 1).

\section{Data sources and searches}

We will use the MEDLINE online database (from 1 January 1980 to 30 June 2015), EMBASE, Web of Science and the Cochrane Database of Systematic Reviews to identify selected studies that evaluated the determinants of obesity including the effect of high versus low carbohydrate diets. In addition, we will evaluate web-based studies that were not published (eg, reports or unpublished theses). Unpublished or so-called 'grey' literature will include reports or documents produced by academics, government and business or industry not controlled by commercial publishers, and will include print as well as electronic formats. Specialised databases containing grey literature, for example, Zetoc (http://zetoc.mimas.ac. $\mathrm{uk} /$ ), which indexes conference proceedings, and Google as well as Google Scholar, will be searched. In addition, registers of ongoing studies, such as the ISRCTN registry (http://www.isrctn.com/), Current Controlled Trials (http://www.controlled-trials.com) and Trials Central (http://www.trialscentral.org), will also be searched. The following keywords or medical subject headings on MEDLINE will be used: ('Diet' or 'lowcarbohydrate diet' or 'low sugar diet' or 'diet, carbohydrate restricted' or 'complex carbohydrates' or 'refined carbohydrates' or 'sugar' or 'sugar sweetened beverages' or 'fat or dietary fibre' or 'protein intake' or 'total carbohydrate intake' or 'total calorie intake') AND ('body mass index' or 'BMI' or 'waist circumference' or 'obesity' or 'blood glucose' or 'fat mass' or 'free fat mass'). The selected studies will include randomised controlled trials (RCTs), including cluster RCTs, controlled (nonrandomised) clinical trials (CCTs) or cluster trials, interrupted time series (ITS) studies with at least three data points before and after the intervention, ${ }^{36}$ controlled before-after (CBA) studies, prospective and retrospective comparative cohort studies and case-control or nested case-control studies or cross-sectional studies. We will exclude case series and case reports.

\section{Study screening and selection}

We will include studies examining the general adult human population or healthy adult humans (18 years or older). We will also include studies on people who are overweight or obese, but will otherwise exclude (account for) studies of populations restricted to specific diseases, conditions or metabolic disorders. Of specific interest are general population studies that have investigated prevalence of obesity in relation to detailed dietary intake. ${ }^{22}$ Of interest also may be interventions addressing a reduction in body weight, such as the impact of specific diets that have been developed to reduce BMI, overweight, obesity or fat mass. This would involve studies that compare the effect of different diets and their impact on comparative groups, for example, one group that is subject to a high carbohydrate diet versus another group on a high fat diet. In addition to direct consumption studies, we will also consider interventions that influence consumption, such as educational or policy interventions. Non-specific or multifaceted behavioural, educational, or policy interventions may also be included, subject to the level of evidence that exists for the aforementioned interventions/exposures. We will classify carbohydrates according to the following broad categories:

- Sugars-glucose, fructose, galactose, sucrose, lactose, maltose;

- Starches-homopolysaccharides, including vegetable, fruit, roots, cereals, whole grain, refined grain, roots, tubers;

- Dietary fibre-resistant starch, soluble fibre, insoluble fibre.

\section{Study selection}

Two authors will independently screen study titles and abstracts for potential eligibility. Screening questions will be developed and pilot tested with a subset of records before implementation. Full texts of potentially eligible studies will be retrieved and two authors will independently apply inclusion criteria to identify appropriate studies to be included in this review. Disagreement will be assessed using $\kappa$ statistics and will be resolved through discussion; a third reviewer will be involved if necessary. We will provide a table with characteristics of included studies and another table of excluded studies with reasons for their exclusion, in our published final review. 
Appraisal of the quality of included studies

Two reviewers are contents experts and one reviewer is an experienced biostatistician/epidemiologist. The contents experts will only assess potential publications with respect to the appropriateness of the research questions tested. The biostatistician will only evaluate the appropriateness of methods employed.

We will evaluate included studies for quality and bias using an adapted version of the Risk of Bias Tool for Prevalence Studies, developed by Hoy et al..$^{37}$ Assessment of the risk of selection and attrition bias will use the Cochrane guidelines available in Review Manager V.5.3 (http://tech.cochrane.org/revman). Furthermore, the reporting quality of each study will be assessed using the STROBE checklist. ${ }^{38}$ Risk of bias and quality scores will be presented in a table.

Inclusion criteria

- We will include cross-sectional, case-control or cohort studies assessing risk factors for obesity including dietary carbohydrate intake, or enough data to compute these estimates

- Classification of obesity will be based on BMI or visceral obesity (waist circumference)

- We will consider all published and unpublished studies. No language restriction will be applied

Exclusion criteria

- Studies not performed in human participants

- Studies lacking primary data and/or explicit method description

- Studies with major ethical issues

\section{Data extraction and management}

Feedback will be solicited from the research team on the draft list of data variables for extraction. Data extraction forms will be developed and pilot tested in Distiller SR. One person will extract all information. A second person will verify $20 \%$ of studies for general characteristics information and $100 \%$ of studies regarding outcomes data. Disagreements will be resolved by consensus or by a third team member, if needed. Information on the descriptive and quantitative characteristics of studies will include the following: publication details (eg, year of publication, language, publication status), characteristics of study (eg, study design, methods, country, setting, sample size, number of centres if applicable, duration of follow-up, source of funding), characteristics of population (eg, age, gender, ethnicity, co-interventions, information regarding respondent bias or representativeness of the included population), details about the exposure/intervention (eg, type of diet, per cent of total calories obtained from carbohydrate consumption, method of assessing carbohydrate consumption; type of educational or other interventions and description, type of professional delivering intervention).

\section{Data synthesis/analysis}

Data will be analysed using a random-effect meta-analysis model and incorporating a restricted maximum-likelihood variance estimator. Effect measures will be presented as risk ratios or relative risks, or ORs with $95 \%$ CIs. All analyses will be performed using $\mathrm{R}$ software V.3.2.0 or later ( $\mathrm{R}$ Core Team (2015). R: A language and environment for statistical computing. R Foundation for Statistical Computing, Vienna, Austria. URL http://www.R-project. $\mathrm{org} /$ ). The following packages within $\mathrm{R}$ will be utilised for the meta-analyses: 'meta' V.4.2-0 (General Package for Meta-Analysis) and 'metafor' V.1.9-7 (A comprehensive collection of functions with which to conduct meta-analyses). Recent GRADE guidelines will be utilised for preparing summary tables for the primary outcomes. 3940

We will consider using the rho $(r)$ value, a correlation coefficient, for continuous outcome variables. Statistics from individual studies will be converted to an $r$ value before meta-analysing. ${ }^{41}$ The $r$ value can be roughly interpreted as a small $(\mathrm{r}=0.1)$, medium $(\mathrm{r}=0.3)$ or large $(\mathrm{r}=0.5)$ effect size. We plan to transform the pooled $\mathrm{r}$ to another statistic, such as an OR, to aid in interpretation. ${ }^{41}$ For time-to-event data, the HR, which is usually estimated from a Cox proportional hazards model, will be pooled using the generic inverse variance method. ${ }^{42}$

\section{Heterogeneity}

We will assess statistical heterogeneity in our meta-analysis, using the $\mathrm{I}^{2}$ statistic. If the $\mathrm{I}^{2}$ is $>50 \%$, we will regard this as substantial heterogeneity.

\section{Publication bias}

If there are $\geq 10$ studies in the meta-analysis, we will further investigate publication bias using funnel plots and Egger's test. ${ }^{43}$ If asymmetry is present based on visual assessment, we will perform exploratory analyses to investigate and adjust this using trim and/or fill analysis. ${ }^{44}$

\section{Sensitivity analysis}

To further identify potential sources of heterogeneity, we will perform the following subgroup analysis:

1. Type of study design;

2. Type of carbohydrate intake;

3. Type of cancer.

In addition, meta-regression may be performed according to the mean follow-up period of the included studies, for example, pertinent to RCT's or prospective cohorts.

\section{Part 2: obesity and cancer}

\section{Data sources and extraction}

We will use the MEDLINE online database (from 1 January 1980 to 30 June 2015), EMBASE, Web of Science and the Cochrane Database of Systematic Reviews, to identify selected studies that evaluated the relationship between obesity and specific cancers. The following keywords or medical subject headings on PUBMED will be used: ('body mass index' or 'BMI' or 'waist circumference' or 'obesity' or 'blood glucose' or 
'fat mass' or 'free fat mass') AND ('cancer type'). The selected studies will only include published systematic reviews and meta-analyses.

\section{Combining parts 1 and 2 to estimate carbohydrate impact of specific cancer types \\ Development of a multiplicative PAF}

The estimated PAF of obesity ascribed to carbohydrate intake (after adjustment for other dietary intake and physical activity) will be multiplied by the measure of effects associated with obesity and each specific cancer, to estimate a cancer-specific carbohydrate contribution. The PAF will be estimated using conventional population PAF calculations ${ }^{45}$ as well as using decomposition (Shapley) values. ${ }^{46}$

PAF are helpful tools for public health planning ${ }^{45}$ and are often underutilised. PAF estimate the proportion of a health outcome that can be ascribed to a particular risk exposure, ${ }^{45}$ in this case, carbohydrate intake versus obesity. Furthermore, the validity of the PAF approach using adjusted regression coefficients in quantifying the relative importance of different factors against more commonly used decomposition approaches such as Shapley ${ }^{46}$ is also of importance. The magnitude of the contribution from each factor may not be accurately estimated by the regression (eg, residual confounding) leading to potentially overestimated or underestimated PAF estimates. ${ }^{47}$ This is particularly relevant to ecological data that cannot be disaggregated to show discrete exposure to each determinant. Also, variables not classified as a prevalence of exposure (ie, proportion) are problematic, as the PAF cannot be directly calculated using traditional formulae. ${ }^{47}$

The proposed calculation (along with relevant margins of uncertainty, ie, $95 \%$ CIs) will be modelled as follows:

Multiplicative cancer-specific fraction due to high carb = [Meta weighted proportion of obesity attributed to high carbohydrate intake (plus lower and upper bounds)] $\times$ [Meta weighted proportion of specific cancer types attributed to obesity (plus lower and upper 95\% confidence bounds) ]

\section{DISCUSSION}

The linkage between carbohydrates and cancer outcomes continues to be an intense focus of epidemiological research. To date, most studies have attempted to assess the direct relationship either between carbohydrates and specific cancers or between obesity and cancer. However, the quantified influence of high carbohydrate intake on cancer, via its role on obesity, has not been assessed, to the best of our knowledge, using a two stage meta-analytical approach. This study will attempt to address this gap in an area of major importance that also includes a renewed focus on the controversy surrounding dietary options.
The main possible limitations of this review could be the scarcity of studies on the subject. Data presented would, therefore, only be general indicators of the epidemiology of cancer related to high carbohydrate intake as mediated via obesity. Cancer related to high carbohydrate intake in the absence of obesity will not be assessed due to the difficulty of the confounding effect of multiple variables and the lack of literature available to quantify this effect.

\section{Author affiliations}

${ }^{1}$ Discipline of Public Health Medicine, School of Nursing and Public Health, University of KwaZulu-Natal, Durban, KwaZulu-Natal, South Africa

${ }^{2}$ University of KwaZulu-Natal Gastrointestinal Cancer Research Centre (GICRC), Durban, KwaZulu-Natal, South Africa

${ }^{3}$ Faculty of Commerce, University of the Witwatersrand, Johannesburg, South Africa

${ }^{4}$ School of Clinical Medicine, University of KwaZulu-Natal, Durban, KwaZuluNatal, South Africa

${ }^{5}$ South African Medical Research Council, Cape Town, South Africa

${ }^{6}$ Division of Exercise Science and Sports Medicine, University of Cape Town

Contributors BS, KS, CA, TEM and TN drafted the manuscript. All the authors contributed to the development of the selection criteria, the risk of bias assessment strategy and data extraction criteria. BS and KS developed the search strategy. BS provided statistical expertise. All the authors read, provided feedback and approved the final manuscript.

Funding This study is funded through the MRC South Africa (University of KwaZulu-Natal Gastrointestinal Cancer Research Centre (GICRC) (grant number MRC-RFA-CCRC-01-2014). CS is employed by the MRC and therefore now, as a formal member of our cancer research group, had a role in the design and preparation of this manuscript.

Competing interests None declared.

Provenance and peer review Not commissioned; externally peer reviewed.

Open Access This is an Open Access article distributed in accordance with the Creative Commons Attribution Non Commercial (CC BY-NC 4.0) license, which permits others to distribute, remix, adapt, build upon this work noncommercially, and license their derivative works on different terms, provided the original work is properly cited and the use is non-commercial. See: http:// creativecommons.org/licenses/by-nc/4.0/

\section{REFERENCES}

1. Hu T, Mills KT, Yao L, et al. Effects of low-carbohydrate diets versus low-fat diets on metabolic risk factors: a meta-analysis of randomized controlled clinical trials. Am J Epidemiol 2012;176(Suppl 7):S44-54.

2. Torre LA, Bray F, Siegel RL, et al. Global cancer statistics, 2012. CA Cancer J Clin 2015;65:87-108.

3. Parkin DM, Nambooze S, Wabwire-Mangen F, et al. Changing cancer incidence in Kampala, Uganda, 1991-2006. Int J Cancer 2010;126:1187-95.

4. Tesfaye F, Nawi N, Van Minh $\mathrm{H}$, et al. Association between body mass index and blood pressure across three populations in Africa and Asia. J Hum Hypertens 2007;21:28-37.

5. Key T, Spencer E. Carbohydrates and cancer: an overview of the epidemiological evidence. Eur J Clin Nutr 2007;61:S112-21.

6. Gnagnarella P, Gandini S, La Vecchia C, et al. Glycemic index, glycemic load, and cancer risk: a meta-analysis. Am J Clin Nutr 2008;87:1793-801.

7. Aune $\mathrm{D}$, Chan $\mathrm{D}$, Lau $\mathrm{R}$, et al. Carbohydrates, glycemic index, glycemic load, and colorectal cancer risk: a systematic review and meta-analysis of cohort studies. Cancer Causes Control 2012;23:521-35.

8. Aune D, Chan D, Vieira A, et al. Dietary fructose, carbohydrates, glycemic indices and pancreatic cancer risk: a systematic review and meta-analysis of cohort studies. Ann Oncol 2012;23:2536-46.

9. Fedirko V, Lukanova A, Bamia C, et al. Glycemic index, glycemic load, dietary carbohydrate, and dietary fiber intake and risk of liver and biliary tract cancers in Western Europeans. Ann Oncol 2013;24:543-53. 
10. Dobbins M, Decorby K, Choi B. The association between obesity and cancer risk: a meta-analysis of observational studies from 1985 to 2011. ISRN Prev Med 2013;2013:680536.

11. Park M, Je Y, Lee JE. Body mass index and biliary tract disease: a systematic review and meta-analysis of prospective studies. Prev Med 2014;65:13-22.

12. Zhang $\mathrm{Y}$, Liu $\mathrm{H}$, Yang $\mathrm{S}$, et al. Overweight, obesity and endometrial cancer risk: results from a systematic review and meta-analysis. Int $J$ Biol Markers 2013;29:e21-9.

13. Shao C, Bai L-P, Qi Z-Y, et al. Overweight, obesity and meningioma risk: a meta-analysis. PLOS ONE 2014;9:e90167.

14. Joshi RK, Lee S-A. Obesity related adipokines and colorectal cancer: a review and meta-analysis. Asian Pac J Cancer Prev 2014;15:397-405.

15. Barclay AW, Petocz P, McMillan-Price J, et al. Glycemic index, glycemic load, and chronic disease risk-a meta-analysis of observational studies. Am J Clin Nutr 2008;87:627-37.

16. Guevara-Aguirre J, Rosenbloom AL. Obesity, diabetes and cancer: insight into the relationship from a cohort with growth hormone receptor deficiency. Diabetologia 2015;58:37-42.

17. Laguna JC, Alegret M, Roglans N. Simple sugar intake and hepatocellular carcinoma: epidemiological and mechanistic insight. Nutrients 2014;6:5933-54.

18. Lahmann PH, Ibiebele TI, Webb PM, et al. A case-control study of glycemic index, glycemic load and dietary fiber intake and risk of adenocarcinomas and squamous cell carcinomas of the esophagus: the Australian Cancer Study. BMC Cancer 2014;14:877.

19. Bosetti C, Pelucchi C, La Vecchia C. Diet and cancer in Mediterranean countries: carbohydrates and fats. Public Health Nutr 2009;12:1595-600.

20. Jebb SA. Aetiology of obesity. Br Med Bull 1997;53:264-85.

21. Jebb SA. Dietary strategies for the prevention of obesity. Proc Nutr Soc 2005;64:217-27.

22. Jebb SA. Carbohydrates and obesity: from evidence to policy in the UK. Proc Nutr Soc 2015;74:215-20.

23. Nordmann AJ, Nordmann A, Briel M, et al. Effects of low-carbohydrate vs low-fat diets on weight loss and cardiovascular risk factors: a meta-analysis of randomized controlled trials. Arch Intern Med 2006;166:285-93.

24. Hession M, Rolland C, Kulkarni U, et al. Systematic review of randomized controlled trials of low-carbohydrate vs. low-fat low-calorie diets in the management of obesity and its comorbidities. Obes Rev 2009;10:36-50.

25. Santos F, Esteves S, da Costa Pereira A, et al. Systematic review and meta-analysis of clinical trials of the effects of low carbohydrate diets on cardiovascular risk factors. Obes Rev 2012;13:1048-66.

26. Ma Y, Olendzki B, Chiriboga D, et al. Association between dietary carbohydrates and body weight. Am J Epidemiol 2005;161:359-67.

27. Malik VS, Schulze MB, Hu FB. Intake of sugar-sweetened beverages and weight gain: a systematic review. Am J Clin Nutr 2006;84:274-88.

28. Krieger JW, Sitren HS, Daniels MJ, et al. Effects of variation in protein and carbohydrate intake on body mass and composition during energy restriction: a meta-regression. Am J Clin Nutr 2006;83:260-74.

29. Shanmugalingam T, Crawley D, Bosco C, et al. Obesity and cancer: the role of vitamin D. BMC Cancer 2014;14:712.
30. Guh DP, Zhang W, Bansback N, et al. The incidence of co-morbidities related to obesity and overweight: a systematic review and meta-analysis. BMC Public Health 2009;9:88.

31. Wang F, Xu Y. Body mass index and risk of renal cell cancer: a dose-response meta-analysis of published cohort studies. Int $J$ Cancer 2014;135:1673-86.

32. Ioannides S, Barlow P, Elwood J, et al. Effect of obesity on aromatase inhibitor efficacy in postmenopausal, hormone receptor-positive breast cancer: a systematic review. Breast Cancer Res Treat 2014;147:237-48.

33. Castillo JJ, Ingham RR, Reagan $\mathrm{JL}$, et al. Obesity is associated with increased relative risk of diffuse large B-cell lymphoma: a meta-analysis of observational studies. Clin Lymphoma Myeloma Leuk 2014;14:122-30.

34. Ma J, Huang M, Wang L, et al. Obesity and risk of thyroid cancer: evidence from a meta-analysis of 21 observational studies. Med Sci Monit 2015;21:283-91.

35. Moher D, Shamseer L, Clarke M, et al. Preferred reporting items for systematic review and meta-analysis protocols (PRISMA-P) 2015 statement. Syst Rev 2015;4:1.

36. Bero L, Grilli R, Grimshaw J, et al. Cochrane effective practice and organisation of care group. The Cochrane Library, 2007.

37. Hoy D, Brooks $P$, Woolf $A$, et al. Assessing risk of bias in prevalence studies: modification of an existing tool and evidence of interrater agreement. J Clin Epidemiol 2012;65:934-9.

38. Von Elm E, Altman DG, Egger M, et al. The Strengthening the Reporting of Observational Studies in Epidemiology (STROBE) statement: guidelines for reporting observational studies. Prev Med 2007:45:247-51.

39. Guyatt GH, Oxman AD, Santesso N, et al. GRADE guidelines: 12. Preparing Summary of Findings tables-binary outcomes. J Clin Epidemiol 2013;66:158-72.

40. Guyatt GH, Thorlund K, Oxman AD, et al. GRADE guidelines: 13 . Preparing Summary of Findings tables and evidence profiles-continuous outcomes. J Clin Epidemiol 2013;66: 173-83.

41. Rosenthal R, Rosnow RL, Rubin DB. Contrasts and effect sizes in behavioral research: a correlational approach. Cambridge University Press, 2000.

42. Fu R, Gartlehner G, Grant M, et al. Conducting quantitative synthesis when comparing medical interventions: AHRQ and the Effective Health Care Program. J Clin Epidemiol 2011;64:1187-97.

43. Egger M, Smith GD, Schneider M, et al. Bias in meta-analysis detected by a simple, graphical test. BMJ 1997;315:629-34

44. Duval S, Tweedie R. Trim and fill: a simple funnel-plot-based method of testing and adjusting for publication bias in meta-analysis. Biometrics 2000;56:455-63.

45. Northridge ME. Public health methods-attributable risk as a link between causality and public health action. Am J Public Health 1995;85:1202-4.

46. Shorrocks AF. Decomposition procedures for distributional analysis: a unified framework based on the Shapley value. J Econ Inequality 2013;11:99-126.

47. Rockhill B, Newman B, Weinberg C. Use and misuse of population attributable fractions. Am J Public Health 1998;88:15-19. 